\title{
SUPPORTING INFORMATION: \\ Single-Crystalline Monolayer Graphene Wafer on Dielectric Substrate of SiON without Metal \\ Catalysts
}

Bong Gyu Shin ${ }^{1,6,7, *}$, Dae Hwan Boo ${ }^{4}$, Bumsub Song ${ }^{1,2}$, Sunam Jeon ${ }^{1,2}$, Minwoo Kim ${ }^{4}$, Sangwoo Park ${ }^{4}$, Eun Soo An ${ }^{5}$, Jun Sung Kim ${ }^{5}$, Young Jae Song ${ }^{1,3,4,8, *}$, and Young Hee Lee $e^{1,2,3, *}$

${ }^{1}$ Center for Integrated Nanostructure Physics (CINAP), Institute for Basic Science (IBS), Sungkyunkwan University, Suwon 16419, Republic of Korea.

${ }^{2}$ Department of Energy Science, Sungkyunkwan University, Suwon 16419, Republic of Korea.

${ }^{3}$ Department of Physics, Sungkyunkwan University, Suwon 16419, Republic of Korea.

${ }^{4}$ Sungkyunkwan Advanced Institute of Nanotechnology (SAINT), Sungkyunkwan University, Suwon 16419, Republic of Korea.

${ }^{5}$ Department of Physics, Pohang University of Science and Technology, Pohang 37673, Republic of Korea

${ }^{6}$ Center for Quantum Nanoscience (QNS), Institute for Basic Science (IBS), Seoul 03760, Republic of Korea

${ }^{7}$ Department of Physics, Ewha Womans University, Seoul 03760, Republic of Korea

${ }^{8}$ Department of Nano Engineering, Sungkyunkwan University (SKKU), Suwon 16419, Republic of Korea

*Correspondence to: shinbone@ skku.edu (B.G.S.), yjsong@skku.edu (Y.J.S.), leeyoung@skku.edu (Y.H.L.) 


\section{Methods}

Synthesis of SiON-SiC(0001). A SiC substrate was cleaned by acetone and isopropyl alcohol (IPA) subsequently, before putting into a vacuum furnace chamber. Hydrogen etching was performed under 760 Torr of $\mathrm{H}_{2}$ gas at $1550^{\circ} \mathrm{C}$ for $20 \mathrm{~min}$. The Joule heating was required for $\mathrm{SiON}$ growth due to the limitation of power for high temperatures ranged of 1100 to $1600^{\circ} \mathrm{C}$, not for graphene growth at $\sim 1000^{\circ} \mathrm{C}$. Graphene growth on $\mathrm{SiON}-\mathrm{SiC}$ was confirmed both cases of Joule heating and conventional CVD furnace cases. After hydrogen etching, nitrogen gas of 760 Torr was replaced with hydrogen gas, while temperature of the sample was kept at $1550^{\circ} \mathrm{C}$. Annealing under $\mathrm{N}_{2}$ gas of 760 Torr was carried out for 20 min. Note that temperature range of 1350 to $1600^{\circ} \mathrm{C}$ shows similar quality of $\mathrm{SiON}-\mathrm{SiC}$ and the only difference among temperature conditions is hydrogen etching rate. After cooling to room temperature (RT) under vacuum, the sample was exposed to pure oxygen gas or air for oxidation of the surface. Low energy diffraction (LEED), scanning tunneling microscopy/spectroscopy (STM/S), and X-ray photoemission spectroscopy (XPS) were performed to identify the SiON-layer. LEED of $\mathrm{SiON}-\mathrm{SiC}(0001)$ shows $\sqrt{3} \times \sqrt{3} \mathrm{R} 30^{\circ}$ patterns even up to $1 \mathrm{keV}$ in a good agreement with previous reports. ${ }^{26, \mathrm{~S} 3} \mathrm{STM} / \mathrm{S}$ measurements exhibited a very flat terrace with surface roughness of $\sim 0.012 \mathrm{~nm}$ and a bandgap of $8.3 \mathrm{eV}$. All SiC substrates were N-doped for Joule heating. The Joule heating was required for $\mathrm{SiON}$ growth due to the limitation of power for high temperatures ranged of 1100 to $1600^{\circ} \mathrm{C}$, not for graphene growth at $\sim 1000^{\circ} \mathrm{C}$. Graphene growth on $\mathrm{SiON}-\mathrm{SiC}$ was confirmed both cases of Joule heating and conventional CVD furnace cases. $4 \mathrm{H}-\mathrm{SiC}$ and $6 \mathrm{H}-$ $\mathrm{SiC}$ were both used to grow graphene and an SiON-layer, which did not show significant difference for each case.

Growth of graphene on SiON-SiC(0001). Monolayer graphene on $\mathrm{SiON}-\mathrm{SiC}(0001)$ was grown by chemical vapor deposition (CVD) method in a vacuum furnace chamber without a metal catalyst. After synthesis of $\mathrm{SiON}-\mathrm{SiC}(0001)$, graphene on an $\mathrm{SiON}$ layer was grown at $1000^{\circ} \mathrm{C}$ under $\mathrm{CH}_{4}$ gas pressure of 1.5-20 Torr for 5-120 min (Figure S1). The optimized condition for monolayer graphene growth was $\sim 60$ min growth time under 20 Torr of $\mathrm{CH}_{4}$. For the single-crystalline graphene growth, the quality of an $\mathrm{SiON}$ layer is critical.

Scanning tunneling microscopy and spectroscopy. Scanning tunneling microscopy (STM) 
and spectroscopy (STS) were performed on $\mathrm{SiON}-\mathrm{SiC}(0001)$ and graphene/SiON-SiC(0001) in an ultra-high vacuum (UHV) chamber of $\sim 2 \times 10^{-11}$ Torr by using a commercial STM (Omicron, Germany). The differential conductance $(d I / d V)$ was measured with a conventional lock-in technique by applying a small voltage modulation of $50 \mathrm{mV}_{\mathrm{rms}}$ at $817 \mathrm{~Hz}$. A tungsten tip was electrochemically etched, and electron bombardment was applied to remove the oxide of the tip apex.

Low energy electron diffraction (LEED) measurements. LEED (Specs, Germany) was combined with STM in a UHV system. Position alignments for LEED measurements were conducted by four axes manipulator. Samples were grounded and an electron beam size is about $1 \mathrm{~mm}$. Each LEED image was obtained by a commercial digital camera with the same focus conditions. For a bare $\mathrm{SiON}-\mathrm{SiC}(0001)$, LEED shows $\sqrt{3} \times \sqrt{ } 3 \mathrm{R} 30^{\circ}$ even up to $1 \mathrm{keV}$ corresponding to the penetration depth of $\sim 1 \mathrm{~nm}$ which is comparable to the thickness of SiON-layer.

Raman spectroscopy measurements. Raman spectroscopy measurements (Nanobase, Korea) were performed with a laser of $532 \mathrm{~nm}$ wavelength. Confocal Raman mapping was carried out over the region of $20 \times 20 \mu \mathrm{m}^{2}$ in ambient conditions. A spatial resolution of confocal Raman mapping was $432 \mathrm{~nm}$. Raman mapping images of each peak was obtained by Gaussian fitting of each spectrum. Analysis of Raman spectra were performed via subtraction of $\mathrm{SiON}-\mathrm{SiC}$ reference.

X-ray photoemission spectroscopy (XPS) measurements. XPS measurements (ESCALAB 250Xi with a monochromated Al Ka X-ray source, Thermo Fisher Scientific, UK) were performed in a UHV chamber with a beam size of $650 \mu \mathrm{m}$. The pass energy is $20 \mathrm{eV}$ in the mode of constant analyzer energy (CAE). XPS results were calibrated to the SiC bulk.

Density functional theory (DFT) calculations. DFT calculations with Vienna $a b$ initio Simulation package (VASP) were performed in projector-augmented wave (PAW) pseudopotential with the generalized gradient approximation (GGA) as parameterized by Perdew, Burke, and Ernzerfhof (PBE) for exchange-correlation functional. ${ }^{\text {S4-S6 }}$ DFT calculations with van der Waals interactions were carried out by the non-local van der Waals density functional (vdW-DF) with optimized exchange functional for correlation part as 
optPBE which is effective for silicon dioxide. ${ }^{\mathrm{S} 7, \mathrm{~S} 8}$ The energy cutoff for the plane-wave basis was set up at $250 \mathrm{eV}$. Force and energy criteria for convergence of calculations were 0.02 $\mathrm{eV} / \AA$ for each atom and $1 \times 10^{-6} \mathrm{eV}$, respectively. The Gamma point was used for k-point sampling. The calculated bandgap with PBE were fitted with previous theoretical results by a scissoring method, whose features in local density of states were almost identical with previous results. ${ }^{\mathrm{S} 9} \mathrm{SiON}-\mathrm{SiC}(0001)$ were modeled as a slab geometry with 4 layers of $\mathrm{SiC}$ $(4 \mathrm{H}-\mathrm{SiC})$ and hydrogen atoms were terminated at the bottom SiC layer for mimicry of a bulk state. The unitcell size corresponds to the $4 \sqrt{3} \times 4 \sqrt{3} \mathrm{R} 30^{\circ}$ of $\mathrm{SiC}(0001)$ with a vacuum region of $\sim 19 \AA$. The adsorption energy ( $\left.\mathrm{E}_{\mathrm{ad}}\right)$ of precursor molecules on SiON-SiC(0001) surface was calculated by the equation; $E_{a d}=\left(E_{S i O N}+E_{\text {precursor }}\right)-E_{\text {precursor/SiON }}$, where $E_{S i O N}, E_{\text {precursor }}$, and $\mathrm{E}_{\text {precursor/SiON }}$ are energies of $\mathrm{SiON}-\mathrm{SiC}(0001)$, precursor molecule, and precursor molecule/SiON-SiC(0001), respectively. For band structure of graphene/SiON-SiC(0001), we choose a minimum unitcell as $\sqrt{3} \times \sqrt{3} \mathrm{R} 30^{\circ}$ due to heavy cost of larger supercell, which is known to capture the main features of electronic structures of graphene on SiC substrate. ${ }^{\mathrm{S} 11}$ 


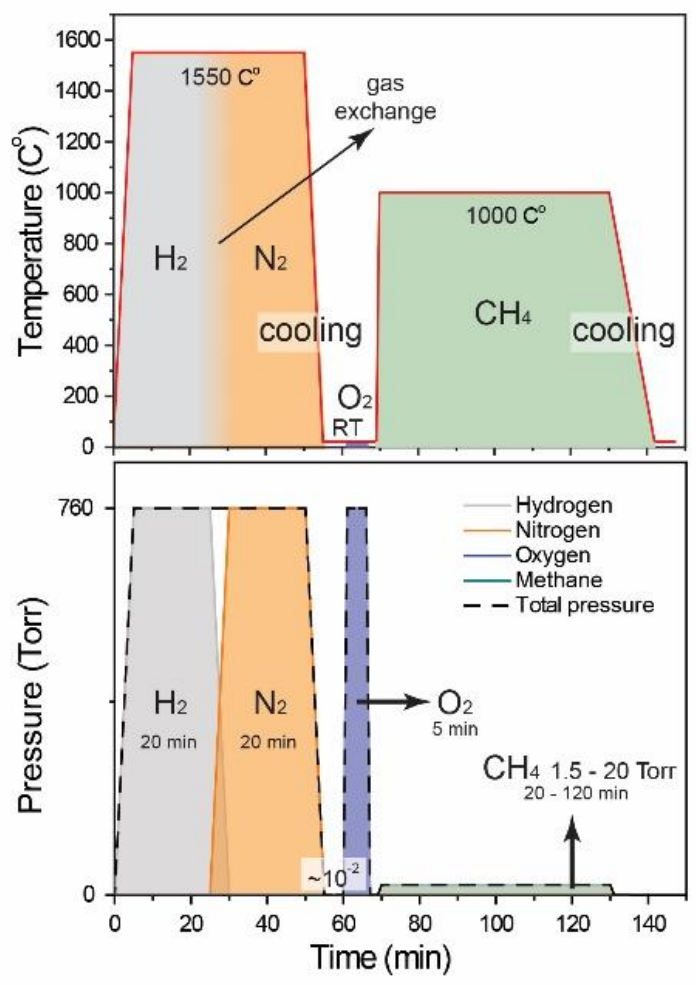

Figure S1 | Growth conditions of SiON and Graphene/SiON. Temperature of a sample (upper plot) and partial pressure of gases (lower plot) as a function of time. An SiON layer on $\mathrm{SiC}$ was synthesized by exposure of oxygen gas at room temperature (RT) after annealing at $1550{ }^{\circ} \mathrm{C}$ under nitrogen gas of 760 Torr. All SiC samples were preliminarily etched by hydrogen gas of 760 Torr at $1550{ }^{\circ} \mathrm{C}$. Gas replacement from hydrogen to nitrogen was carried out subsequently with keeping temperature of the sample. Finally, graphene on an $\mathrm{SiON}$ layer was grown at $1000{ }^{\circ} \mathrm{C}$ under 20 Torr $\mathrm{CH}_{4}$ pressure for $120 \mathrm{~min}$. Slopes in the schematic plots is not in scale. 


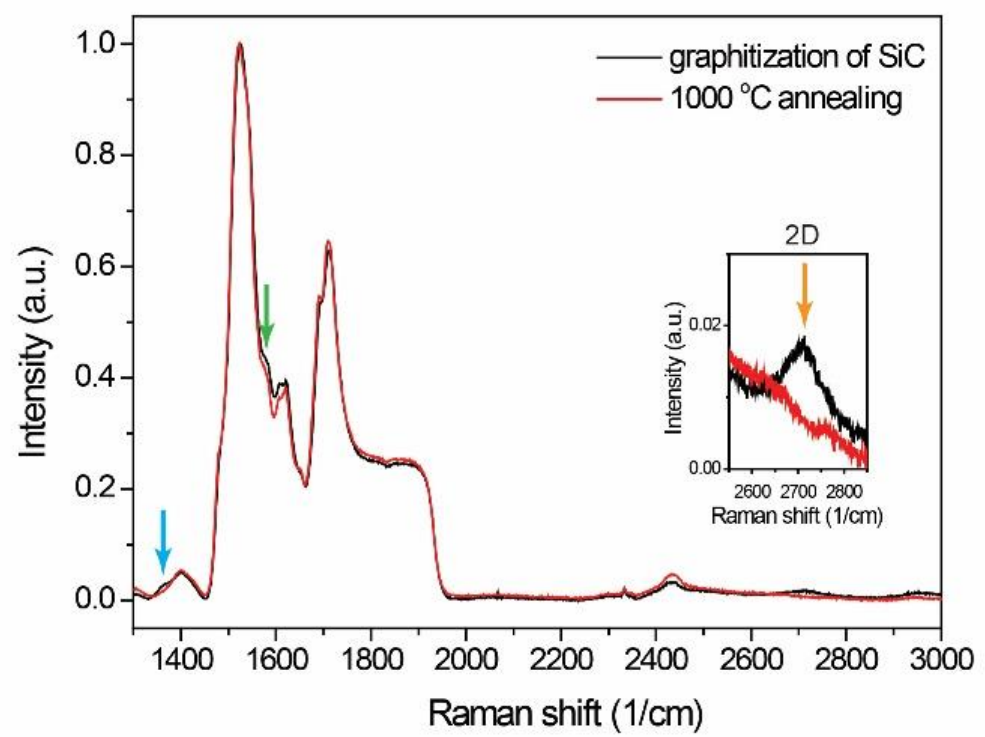

Figure S2 | Annealing effect without $\mathrm{CH}_{4}$ at $1000{ }^{\circ} \mathbf{C}$. Annealing of $\mathrm{SiON}-\mathrm{SiC}(0001)$ at $1000{ }^{\circ} \mathrm{C}$ under pressure of $\sim 10^{-6}$ Torr without $\mathrm{CH}_{4}$ did not show Raman 2D peak or any changes in Raman spectrum, which means $\mathrm{SiON}-\mathrm{SiC}$ was intact during annealing which is in a good agreement with XPS results. (Figure S3) The thermally-decomposed graphitization of $\mathrm{SiC}$ surface $\left(6 \sqrt{3} \times 6 \sqrt{3} \mathrm{R} 30^{\circ}\right.$ at $\sim 1200{ }^{\circ} \mathrm{C}$ in UHV) shows a clear evidence of 2D peak in Raman spectrum, which indicated by orange arrow. G and D peak positions also exhibited enhancement of Raman intensities as indicated by green and blue arrows, respectively. These systematic experiments support that the graphene could be grown on $\mathrm{SiON}-\mathrm{SiC}(0001)$ only when $\mathrm{CH}_{4}$ is supplied at $1000{ }^{\circ} \mathrm{C}$ as shown in Figure 2 in the manuscript.
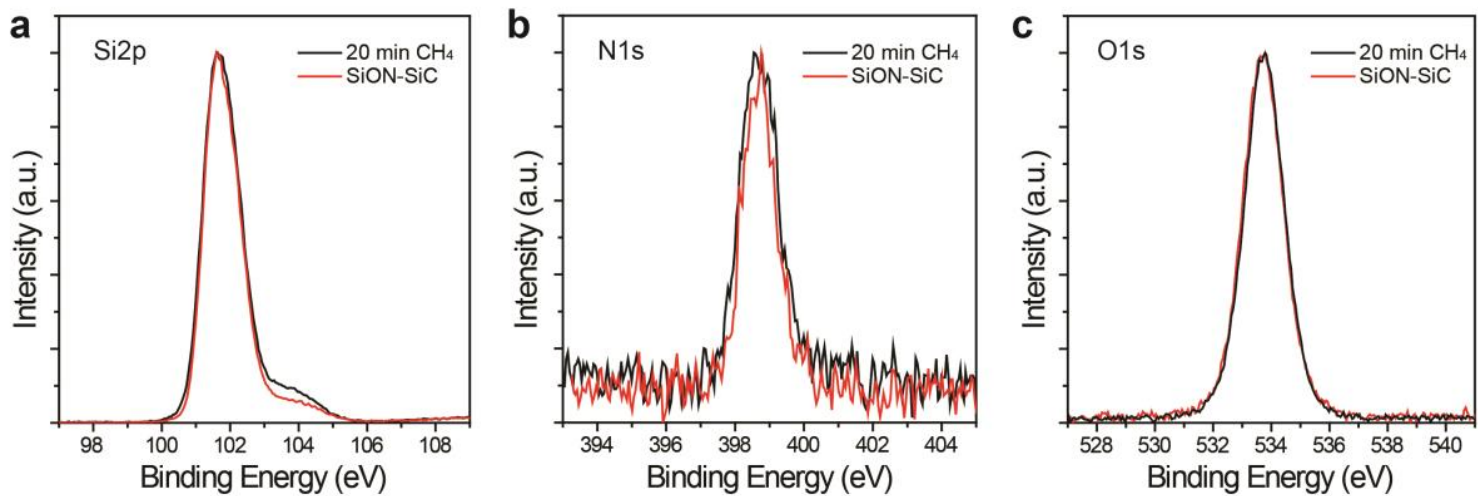

Figure S3 | Before and after growth of graphene on SiON-SiC(0001). The X-ray photoemission spectroscopy (XPS) results show Si2p (a), N1s (b), and O1s (c), respectively. The binding energies in a bare SiON-SiC(0001) were $532.2 \mathrm{eV}$ for $\mathrm{O} 1 \mathrm{~s}$ and 397.5 for N1s, respectively, which is in a good agreement with previous reports. ${ }^{26}$ After monolayer graphene growth, XPS results of O1s $(532.4 \mathrm{eV})$ and N1s $(397.8 \mathrm{eV})$ did not show significant changes, which means that SiON-layer was intact during monolayer graphene growth at $1000{ }^{\circ} \mathrm{C}$. Only XPS of $\mathrm{Si} 2 \mathrm{p}$ (101.7 eV for bulk $\mathrm{SiC}$ ) exhibited relative weak enhancement for topmost configurations of $\mathrm{Si}-\mathrm{N}_{3} \mathrm{O}(103.5$ $\mathrm{eV})$ and $\mathrm{Si}_{-} \mathrm{O}_{4}(104.3 \mathrm{eV})$ compared to that in bulk SiC. In addition, hydrogen-etched $\mathrm{SiC}$ samples without the nitrogen annealing show no peak of N1s in XPS results. 


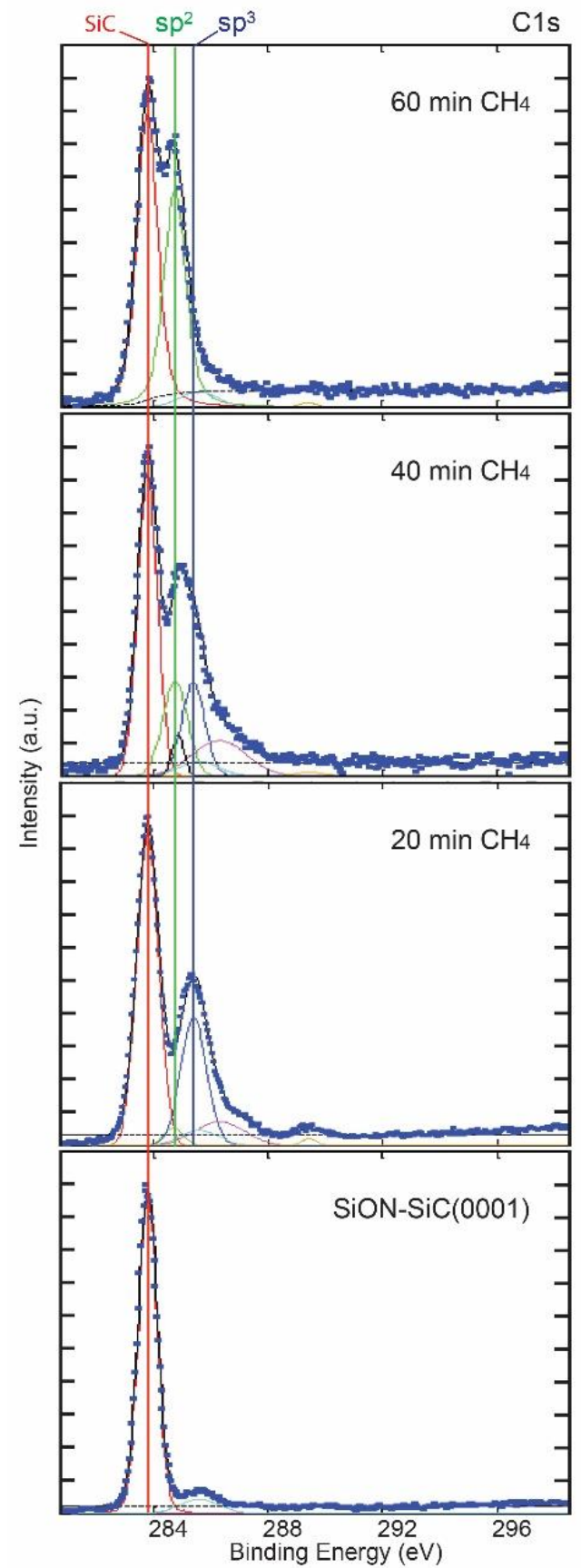

Figure S4 | Growth-time dependency of C1s in XPS. The C atoms in $\mathrm{sp}^{2}$ and $\mathrm{sp}^{3}$ states are plotted in green and blue colors, respectively. As increasing the growth time, $\mathrm{sp}^{2} \mathrm{C}$ atoms became dominant than $\mathrm{sp}^{3} \mathrm{C}$ atoms. Intermediate $\mathrm{C}$ atoms between $\mathrm{sp}^{2}$ and $\mathrm{sp}^{3}$ appeared at growth time of $20 \mathrm{~min}$, which is plotted in a black color. After growth time of $60 \mathrm{~min}$, the peak of $\mathrm{sp}^{3} \mathrm{C}$ atoms disappeared eventually, and only $\mathrm{sp}^{2} \mathrm{C}$ atoms were exhibited. The SiC-related peaks in XPS results were plotted in a red color. The XPS result of SiON-SiC $(0001)$ was analyzed as a reference during the analysis of XPS results. For growth time of below 40 min, the peaks of $286.4 \mathrm{eV}$ (C-OH and $\mathrm{C}-\mathrm{O}$ groups) and $289.4 \mathrm{eV}(\mathrm{COOH})$ were expected by the exposure to air which induces adsorption of oxygenated species at the edges or defects of partially covered graphene flakes on SiON-SiC. ${ }^{\mathrm{S1}, \mathrm{S} 2}$ After growth of fully-covered graphene, the peak related with oxygenated species became negligible because adsorption sites such as defects or edges of flakes are decreasing. 


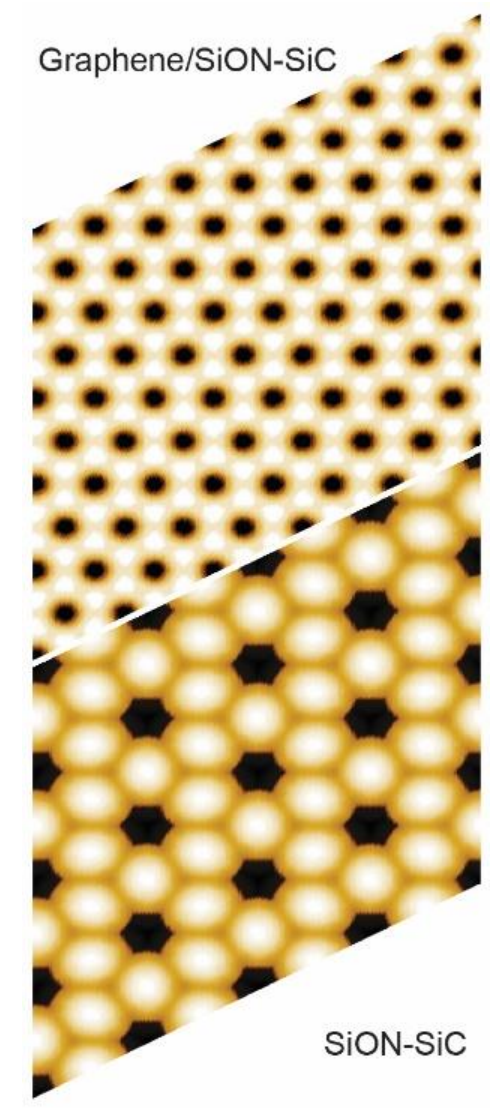

Figure S5 | STM simulation images of bare SiON-SiC(0001) and graphene/SiON-SiC(0001). Upper and lower images are STM simulations of graphene/SiON-SiC(0001) and SiON-SiC(0001), respectively. Size of each image corresponds to $4 \sqrt{3} \times 4 \sqrt{3} \mathrm{R} 30^{\circ}$ of $\mathrm{SiC}(0001)$. STM simulation of graphene/SiON-SiC(0001) do not exhibit Moiré patterns. Sample bias of a bare SiON-SiC in STM simulations is set to $2 \mathrm{eV}$ lower than the valence band edge, which is the same condition of STM image in Figure 3.
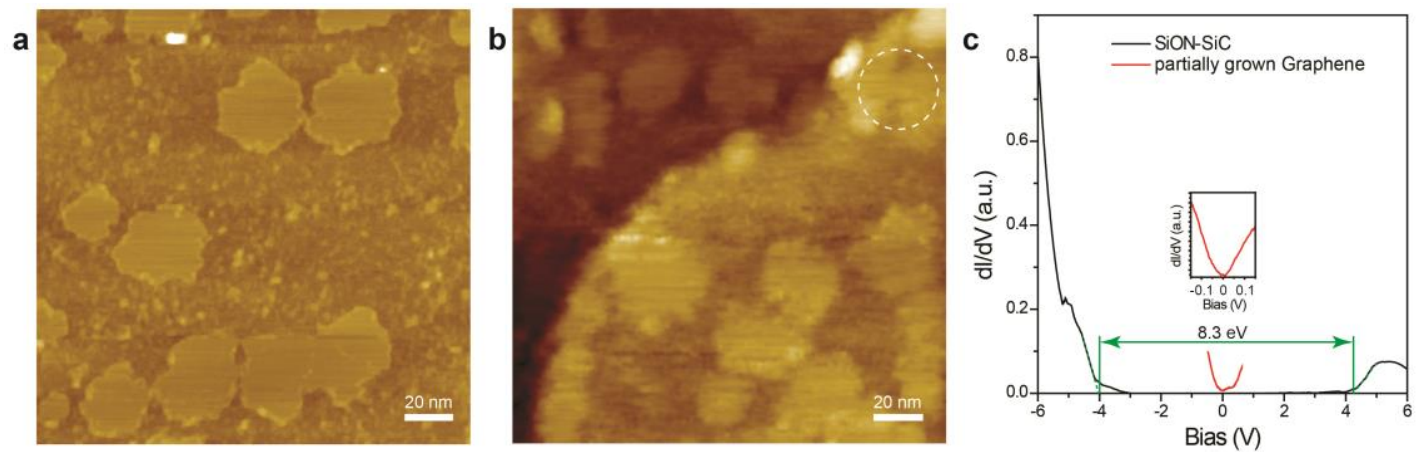

Figure S6 | Partial growth of monolayer graphene on SiON-SiC(0001). (a,b) STM images show that monolayer graphene was partially grown on the surface of SiON-SiC after growth time of each; 20 min (a) and $40 \mathrm{~min}$ (b). A white-dashed circle in (b) indicates the merging of two flakes. Surface coverages of graphene flakes in STM images were evaluated as $\sim 22 \%$ for $20 \mathrm{~min}$ and $\sim 44 \%$ for $40 \mathrm{~min}$, respectively. The density of small particles in 20 min case was higher than that in 40 min case, which was correlated with $\mathrm{sp}^{3} \mathrm{C}$ atoms of XPS results. (Figures 2d and S4) (c) STS results of graphene flakes and SiON-SiC substrates. Apparently, an $\mathrm{SiON}-\mathrm{SiC}$ substrate exhibited a large bandgap of $8.3 \mathrm{eV}$, and graphene shows a Dirac point matching with a Fermi level. This sample was measured without additional thermal annealing. 

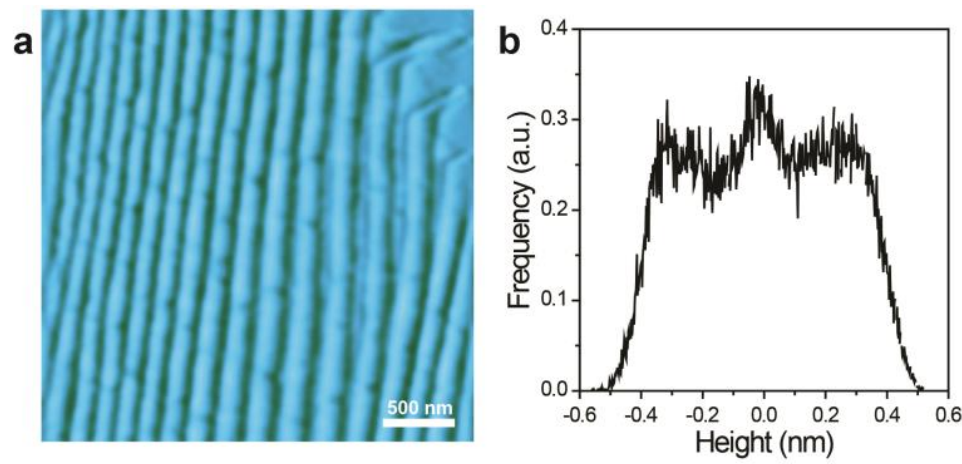

Figure S7 | Surface roughness of SiON-SiC(0001). (a) AFM image of $\mathrm{SiON}-\mathrm{SiC}(0001)$ over $3 \times 3 \mu \mathrm{m}^{2}$. Atomic steps were observed. (b) Histogram of height of (a). Height variation is within $\sim 1 \mathrm{~nm}$. An overall root-meansquare (RMS) surface roughness is $0.24 \mathrm{~nm}$ over $3 \times 3 \mu \mathrm{m}^{2}$ due to the atomic steps on the surface. Terraces exhibited RMS surface roughness of $0.015 \mathrm{~nm}$.
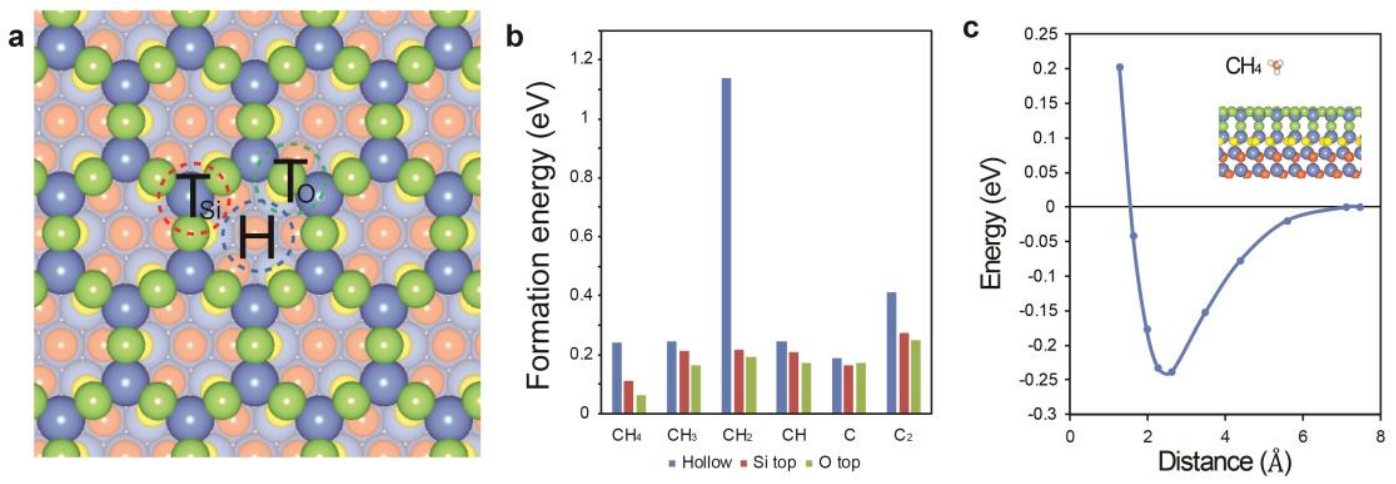

Figure S8 | DFT studies for adsorption configurations of precursors on $\mathrm{SiON}-\mathrm{SiC}(0001)$. (a) Adsorption sites on $\mathrm{SiON}-\mathrm{SiC}(0001)$ were remarked in the top view; a hollow $(\mathrm{H})$, a silicon-top $\left(\mathrm{T}_{\mathrm{Si}}\right)$, and an oxygen-top $\left(\mathrm{T}_{\mathrm{O}}\right)$ sites. Blue and green balls indicate oxygen and silicon atoms, respectively. (b) Adsorption energies of precursors. The average value of all calculated precursors is $-259.1 \mathrm{meV}$. All calculated precursor molecules preferred a $\mathrm{H}$ site. (c) Surface potential energy of $\mathrm{CH}_{4}$ along the perpendicular direction to the surface at a $\mathrm{H}$ site. A distance is calculated between the carbon atom of $\mathrm{CH}_{4}$ and an $\mathrm{SiON}-\mathrm{SiC}$ surface.
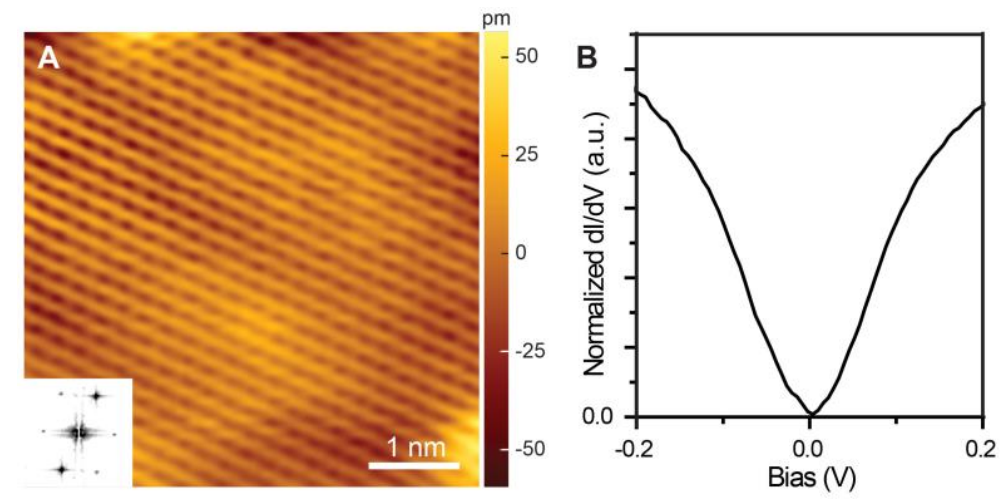

Figure S9 | Transferred Cu-CVD graphene on SiON-SiC(0001). (a) STM image of transferred Cu-CVD graphene on SiON-SiC(0001). Inset is FFT of the STM image. (b) STS result in (a) at $78 \mathrm{~K}$. Even for the transferred graphene on SiON-SiC(0001), the Dirac point is located at a Fermi level, and V-shape STS results are observed. Neutrality of $\mathrm{SiON}-\mathrm{SiC}(0001)$ is intrinsic properties of the substrate, which is not changed during the growth procedure of graphene. 


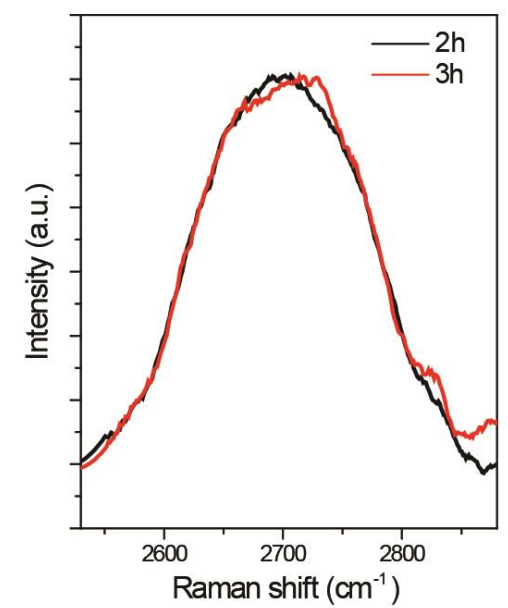

Figure S10 | Self-limiting growth of graphene on SiON-SiC(0001). Raman intensities for 2D peaks of graphene over growth time of 1 hour did not show significant difference.

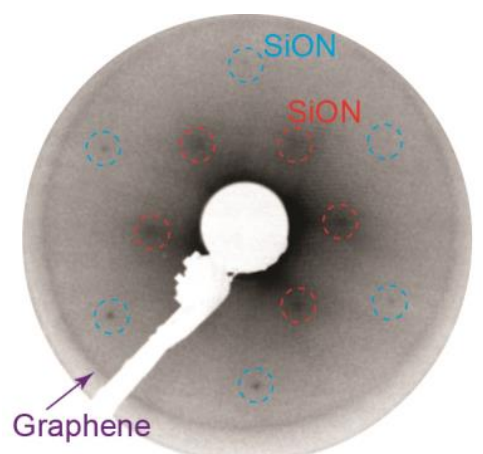

Figure S11 | High pressure effect for graphene growth on SiON-SiC(0001). LEED of graphene grown on $\mathrm{SiON}$ under high pressure of 200 Torr $\mathrm{CH}_{4}(20 \mathrm{~min})$ shows ring pattern which indicates randomness of orientation of graphene without self-limiting monolayer growth. This provides an additional clue for criteria of $\mathrm{SiON}$-assisted monolayer growth mechanism. $(60 \mathrm{eV})$
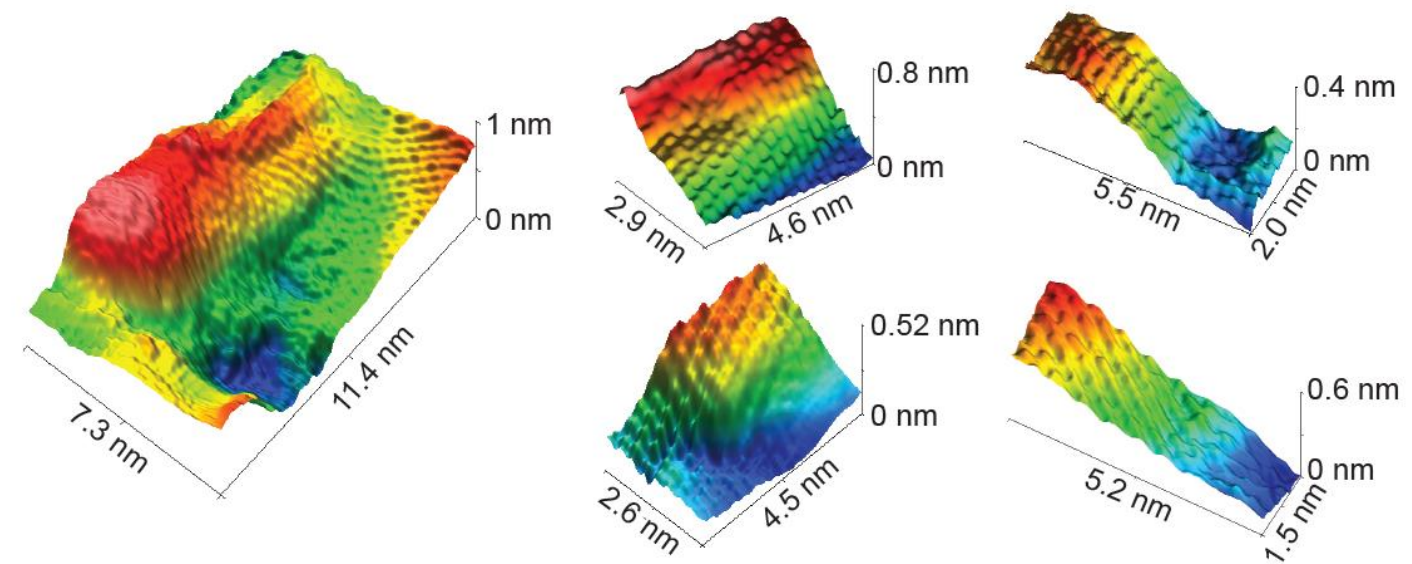

Figure S12 | Continuity of graphene growth on SiON across changes of surface morphology. Each image was obtained from different positions of the sample. Crystalline direction of each coincides each other. (Figures are rotated for better visualization.) 


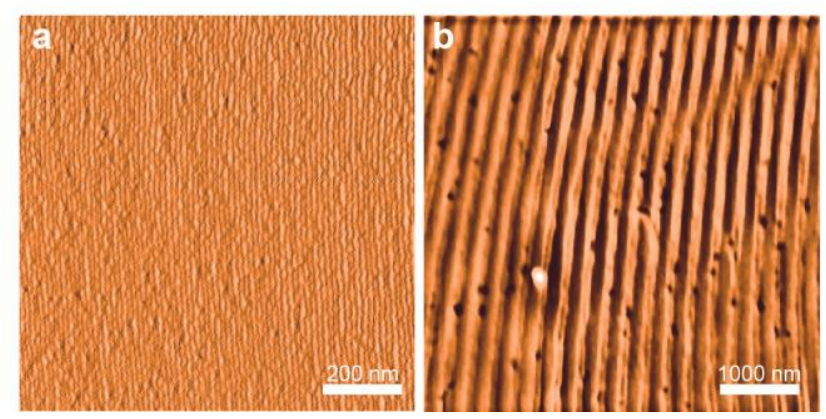

Figure S13 | Comparison between $0^{\circ}$ and $4^{\circ}$ off cut from [0001] after SiON-formation on $\mathrm{SiC}(0001)$. (a) AFM image of $4^{\circ}$ off cut of $\mathrm{SiC}(0001)$ from [0001] after $\mathrm{SiON}$-formation shows regular steps with width of $\sim 20$ $\mathrm{nm}$ and step height of a half atomic unit of [0001] direction. (b) AFM image of $0^{\circ}$ off cut of $\mathrm{SiC}(0001)$ shows wider terrace width of $\sim 200 \mathrm{~nm}$. In addition to, electronic structure and quality of graphene grown on SiON are similar to each case of off-cut angles, $4 \mathrm{H}-$, and $6 \mathrm{H}-\mathrm{SiC}(0001)$, respectively.
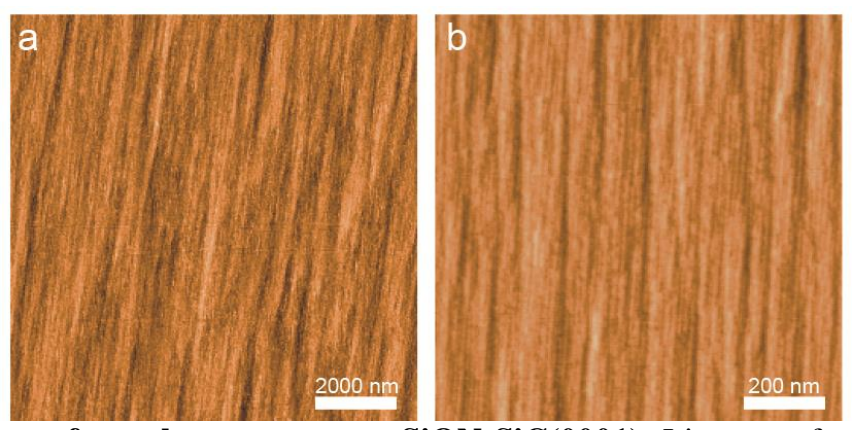

Figure S14 | AFM images of graphene grown on SiON-SiC(0001). Line step features of SiON-SiC(0001) were conserved after graphene growth. Surface morphology was determined by formation of $\mathrm{SiON}-\mathrm{SiC}(0001)$ ( $4^{\circ}$ off cut).

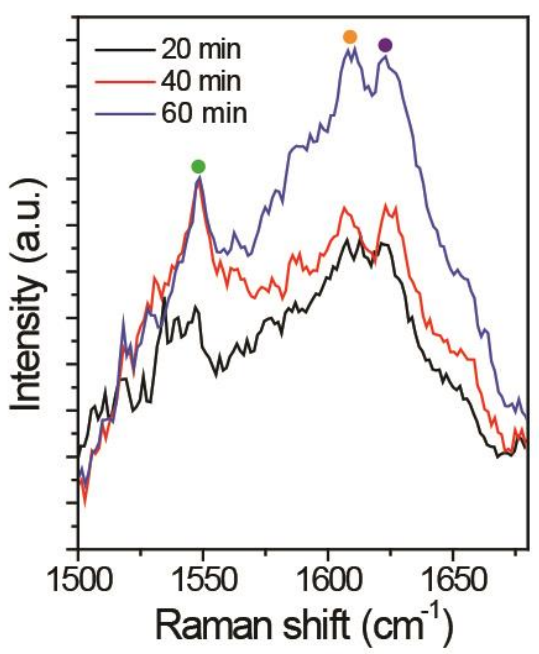

Figure S15 | Raman G peaks and alignment between graphene and SiON. Additional peaks near G peak of $\sim 1600 \mathrm{~cm}^{-1}$ are visible, which can correspond to the alignment within a small angle rotation below $2^{\circ}$, at least, which is very similar to $\mathrm{hBN}$ and graphene with small rotation angle below $2^{\circ} .{ }^{\circ 12}$ In addition to, FWHM of $\sim 40$ $\mathrm{cm}^{-1}$ in $2 \mathrm{D}$ peaks of graphene on SiON is similar to the zero-angle rotation case of $\mathrm{hBN}$ and graphene stacking in the previous study. ${ }^{\mathrm{S12}}$ The peaks of $\sim 1550 \mathrm{~cm}^{-1}$ were assigned as an artefact from a substrate in previous study. ${ }^{\mathrm{S} 12}$ Further study for Raman characteristic features in graphene on SiON should be required. For Figure 4, we used single Gaussian fitting for $\mathrm{G}$ peaks, which is enough to the evidence of existence of monolayer graphene. ${ }^{\text {S12 }}$ 


\begin{tabular}{|c|c|c|c|}
\hline Molecule & Hollow & Si top & O top \\
\hline $\mathrm{CH}_{4}$ & 1.159 & 2.324 & 2.074 \\
\hline $\mathrm{CH}_{3}$ & 2.647 & 2.896 & 3.267 \\
\hline $\mathrm{CH}_{2}$ & 2.076 & 3.270 & 3.116 \\
\hline $\mathrm{CH}$ & 2.037 & 3.076 & 3.251 \\
\hline $\mathrm{C}$ & 0.219 & 1.530 & 2.996 \\
\hline $\mathrm{C}_{2}$ & 2.069 & 2.987 & 3.142 \\
\hline
\end{tabular}

Table S1 | Binding distances of precursors on SiON-SiC(0001). The table shows minimal distances between precursor molecules and an SiON-SiC surface for each adsorption site as shown in Figure S8a. The average value of binding distances for all calculated precursors is $2.45 \AA$, which is smaller than the distance between graphene and $\mathrm{SiON}-\mathrm{SiC}$ surface $(3.28 \AA$ ). The graphene becomes lifted-up to be pseudo-suspended while growing on $\mathrm{SiON}-\mathrm{SiC}(0001)$.

\section{References}

S1. Li, Z.; Wang Y.; Kozbial, A.; Shenoy, G.; Zhou, F.; McGinley, R.; Ireland, P.; Morganstein, B.; Kunkel, A.; Surwade, S. P.; Li, L.; Liu, H. Effect of Airborne Contaminants on the Wettability of Supported Graphene and Graphite. Nat. Mater. 12, 925-931 (2013).

S2. Johra, F. T.; Lee, J.-W.; Jung, W.-G. Facile and Safe Graphene Preparation on Solution Based Platform. J. Ind. Eng. Chem. 20, 2883-2887 (2014).

S3. Shin, B. G. Atomic and Electronic Structures of Two Dimensional Nano-Materials Affected by Substrate Interactions: Graphene, $\mathrm{MoS}_{2}$ and $\mathrm{SiON}-\mathrm{SiC}(0001)$, thesis, Sungkyunkwan University, 91-106 (2014).

S4. Kresse, G.; Furthmüller, Efficiency of Ab-Initio Total Energy Calculations for Metals and Semiconductors Using a Plane-Wave Basis Set. J. Comput. Mater. Sci. 6, 15 (1996).

S5. Kresse, G.; Furthmüller, J. Efficient Iterative Schemes for Ab Initio Total-Energy Calculations Using a Plane-Wave Basis Set. Phys. Rev. B 54, 11169 (1996).

S6. Kresse, G.; Joubert, D. From Ultrasoft Pseudopotentials to the Projector AugmentedWave Method. Phys. Rev. B 59, 1758 (1999).

S7. Perdew, J. P.; Burke, K.; Ernzerhof, M. Generalized Gradient Approximation Made Simple. Phys. Rev. Lett. 77, 3865 (1996).

S8. Klimeš, J.; Bowler, D. R.; Michaelides, A. Chemical Accuracy for the Van der Waals Density Functional. J. Phys.: Cond. Matt. 22, 022201 (2010).

S9. Klimeš, J.; Bowler, D. R.; Michaelides, A. Van der Waals Density Functionals Applied to 
Solids. Phys. Rev. B 83, 195131 (2011).

S10. Shirasawa, T.; Hayashi, K.; Yoshida, H.; Mizuno, S.; Tanaka, S.; Muro, T.; Tamenori, Y.; Harada, Y.; Tokushima, T.; Horikawa, Y.; Kobayashi, E.; Kinoshita, T.; Shin, S.; Takahashi, T.; Ando, Y.; Akagi, K.; Tsuneyuki, S.; Tochihara, H. Atomic-Layer-Resolved Bandgap Structure of an Ultrathin Oxynitride-Silicon Film Epitaxially Grown on 6HSiC(0001). Phys. Rev. B 79, 241301(R) (2009).

S11. Mattausch, A.; Pankratov, O. Ab Initio Study of Graphene on SiC, Phys. Rev. Lett. 99, 076802 (2007).

S12. Eckmann, A.; Park, J.; Yang, H.; Elias, D.; Mayorov, A. S.; Yu, G.; Jalil, R.; Novoselov, K. S.; Gorbachev, R. V.; Lazzeri, M.; Geim, A. K.; Casiraghi, C. Raman Fingerprint of Aligned Graphene/h-BN Superlattices. Nano Lett. 2013, 13, 5242-5246. 